\title{
Analysis and implementation of different topologies of transversal filters in planar technology
}

\author{
D. Cañete Rebenaque, ${ }^{1}$ M. Martínez-Mendoza, ${ }^{1}$ J. L. Gómez-Tornero, ${ }^{1}$ \\ J. Pascual-García, ${ }^{1}$ and A. Álvarez-Melcón ${ }^{1}$
}

Received 21 December 2007; revised 23 April 2008; accepted 20 May 2008; published 30 August 2008.

[1] This paper uses a simple configuration to implement microwave transversal filters based on microstrip technology. The structure is of second order and implements two transmission zeros and two reflection zeros in the frequency response of the filter. The basic configuration consists of input and output ports coupled in a shunt configuration to two printed resonators of different lengths. A modification of the original structure is introduced to allow an additional direct coupling between the source and the load.

Depending on the value and the sign of the coupling terms, different frequency responses can be obtained. In this way, small modifications in the basic structure allow to obtain band-pass or band-stop responses. Following this circuit topology, the paper discusses the possibilities to have transmission zeros in the real or in the complex plane and their effects on the frequency response and on the group delay of the filter. Also, two practical implementations in microstrip technology are proposed, and a third filter implementing group delay equalization is demonstrated. Measured results confirm theoretical predictions and validate the structures for practical applications.

Citation: Cañete Rebenaque, D., M. Martínez-Mendoza, J. L. Gómez-Tornero, J. Pascual-García, and A. Álvarez-Melcón (2008), Analysis and implementation of different topologies of transversal filters in planar technology, Radio Sci., 43, RS4021, doi:10.1029/2007RS003819.

\section{Introduction}

[2] This paper is focused on the implementation of novel filtering microwave structures in planar technology. Because of the need of efficiently use the electromagnetic spectrum in modern communication systems, it is required the development of frequency selective components, exhibiting sharp cut-off slopes [see Williams, 1970]. This need of rejecting certain unwanted frequencies, leads to the development of microwave filters whose insertion loss response exhibits transmission zeros at finite frequencies [see Kurzok, 1966]. These filters, showing pseudo-elliptic responses, normally introduce very large group delay variations, therefore not being suitable in many digital transmissions systems. To solve this problem, several techniques for group delay reduc-

\footnotetext{
${ }^{1}$ Departamento de Tecnologías de la Información y las Comunicaciones, Technical University of Cartagena, UPCT, Cartagena, Murcia, Spain.

Copyright 2008 by the American Geophysical Union. 0048-6604/08/2007RS003819\$11.00
}

tion based on external equalization [Lee et al., 2003], have been proposed in the past. Also, design techniques for phase equalization can directly be applied, as in the study of Cameron and Rhodes [1981].

[3] The method traditionally used for the implementation of transmission zeros at precise frequencies is the introduction of cross-couplings between nonadjacent resonators. An example composed of square open-loop resonators is presented by Hong and Lancaster [2000]. More recently, novel schemes for microwave filters were proposed by Rosenberg and Amari [2003]. In this case, different coupling topologies for microwave filters based on the transversal concept were investigated. The main difference with respect to traditional structures, is that in transversal filters the input signal is coupled at the same time to several resonators. In the original work, several structures of different orders were investigated. The study also included cross couplings between nonadjacent resonators, and couplings between the input port and internal resonators. An extension to in-line filters was presented by Amari and Rosenberg [2004a], where they introduced the concept of internal nonresonating nodes. 


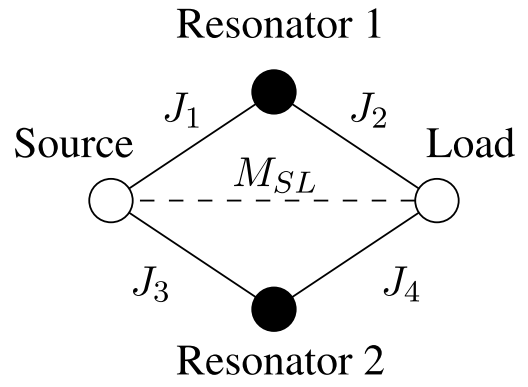

Figure 1. Coupling scheme proposed for the filtering structure.

[4] In the last years, several practical examples of transversal filters implemented in different technologies have been presented. For instance, two simple structures were designed, fabricated and measured by Rebenaque et al. [2004]. Other interesting examples using planar or waveguide technology can be found by Moktaari et al. [2006].

[5] In this paper we will further explore transversal filters in microstrip technology, by implementing the topology known as Modified Doublet (MD) (shown in Figure 1). In this figure, solid line represents coupling between input/output ports and resonators, while dashed line represents coupling between the ports $\left(M_{\mathrm{SL}}\right)$. The coupling scheme is similar to the one introduced by Rosenberg and Amari [2003], but now a direct coupling between input and output ports is introduced, as shown by Amari and Rosenberg [2003]. The advantage of the structure proposed, is that the additional direct coupling introduces a new transmission zero in the insertion loss response of the filter. Therefore two transmission zeros can be obtained for maximum selectivity above and below the passband.

[6] First, it is presented a compact microstrip configuration that implements the coupling scheme shown in Figure 1. The resonators used in the practical implementation are the same as those introduced by Rebenaque et $a l$. [2004]. They consist of an open-loop resonator and of a short-circuited T-shaped stub. We show that by changing the coupling signs appropriately, the positions of the transmission zeros can be conveniently controlled. In addition, we present a discussion on the influence of the position of these transmission zeros, both in the frequency response and in the phase equalization of transversal filters. Finally, a dual frequency response of the above filter can be obtained, just by changing the direct coupling sign $\left(M_{\mathrm{SL}}\right)$ between the ports. Following this idea, we present a novel structure that implements a band-stop response, using the same basic topology and coupling scheme as before (Figure 1). The resonator topologies employed in this second structure are the same open-loop and T-shaped stub used before. Only the original capacitive coupling between the ports is changed in sign, and it is transformed into an inductive type coupling. It is shown in this paper that the change in sign of this direct coupling $\left(M_{\mathrm{SL}}\right)$ transforms the bandpass frequency response into a dual stop-band frequency response.

[7] In addition to the novel implementation of bandstop filters using a transversal topology, an important novel aspect of the work is the practical demonstration of complex transmission zeros using these microstrip structures. Also, this is the first time that an explanation of these microstrip structures, following the transversal filter concept [Cameron, 2003] is presented, together with supporting coupling matrices. Finally, a rigorous study on the direct coupling term $M_{\mathrm{SL}}$, that can be achieved by bending together the input/output ports, is for the first time presented. The introduction of this direct coupling element will allow the implementation of a

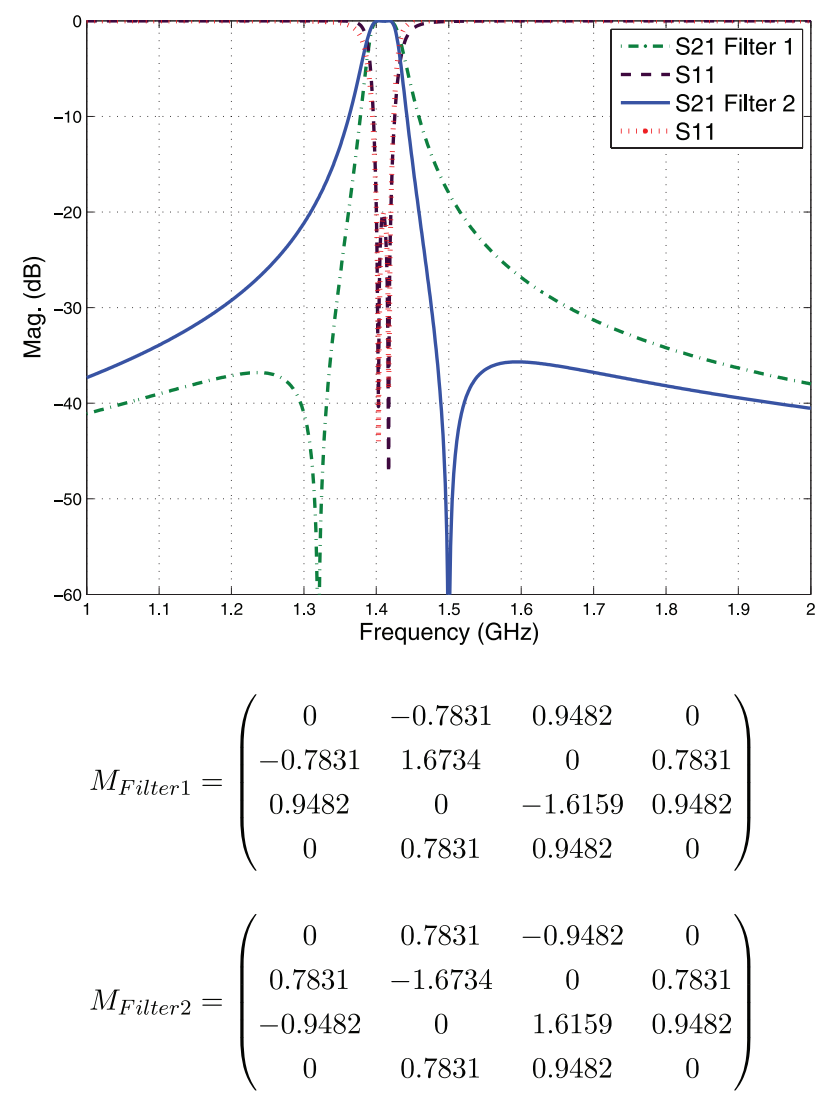

Figure 2. S-parameters of transversal filters without input-output coupling. Examples of transmission zeros located below (Filter 1) or above (Filter 2) the passband. The coupling matrices of the filters are shown for reference. 


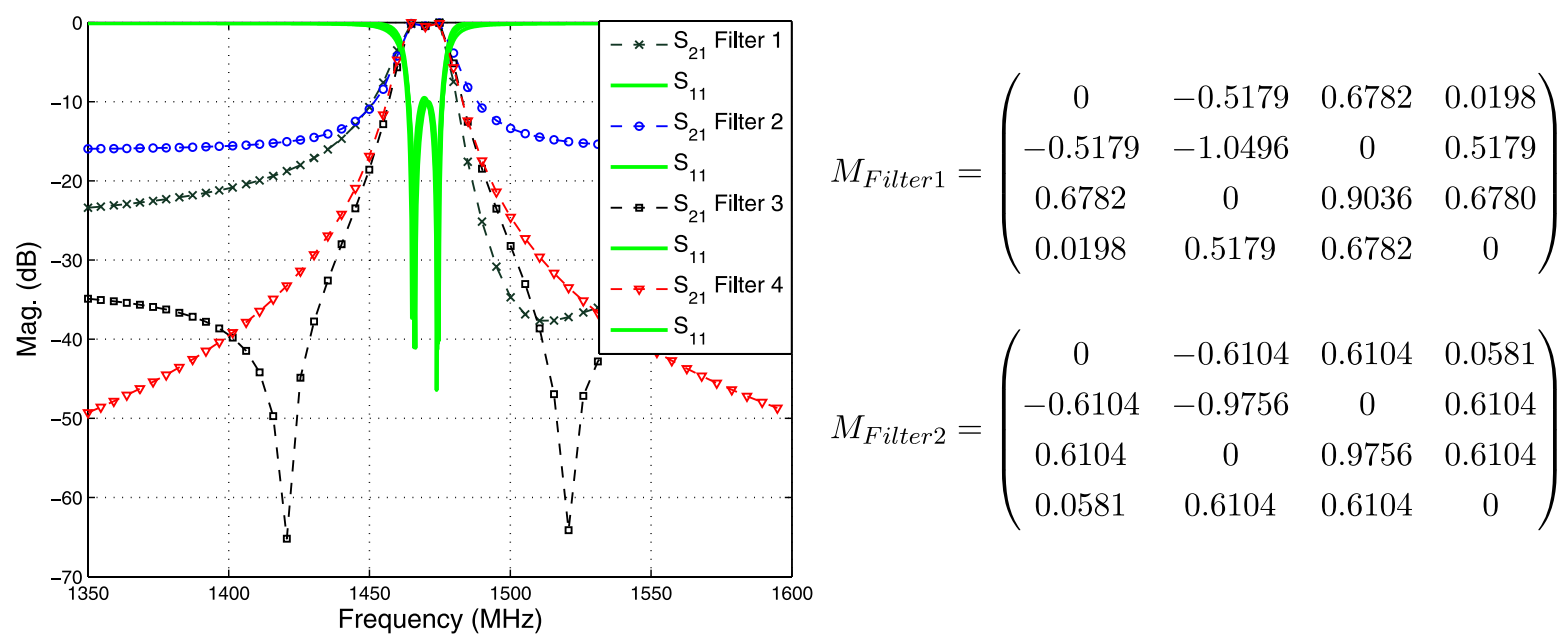

(a)

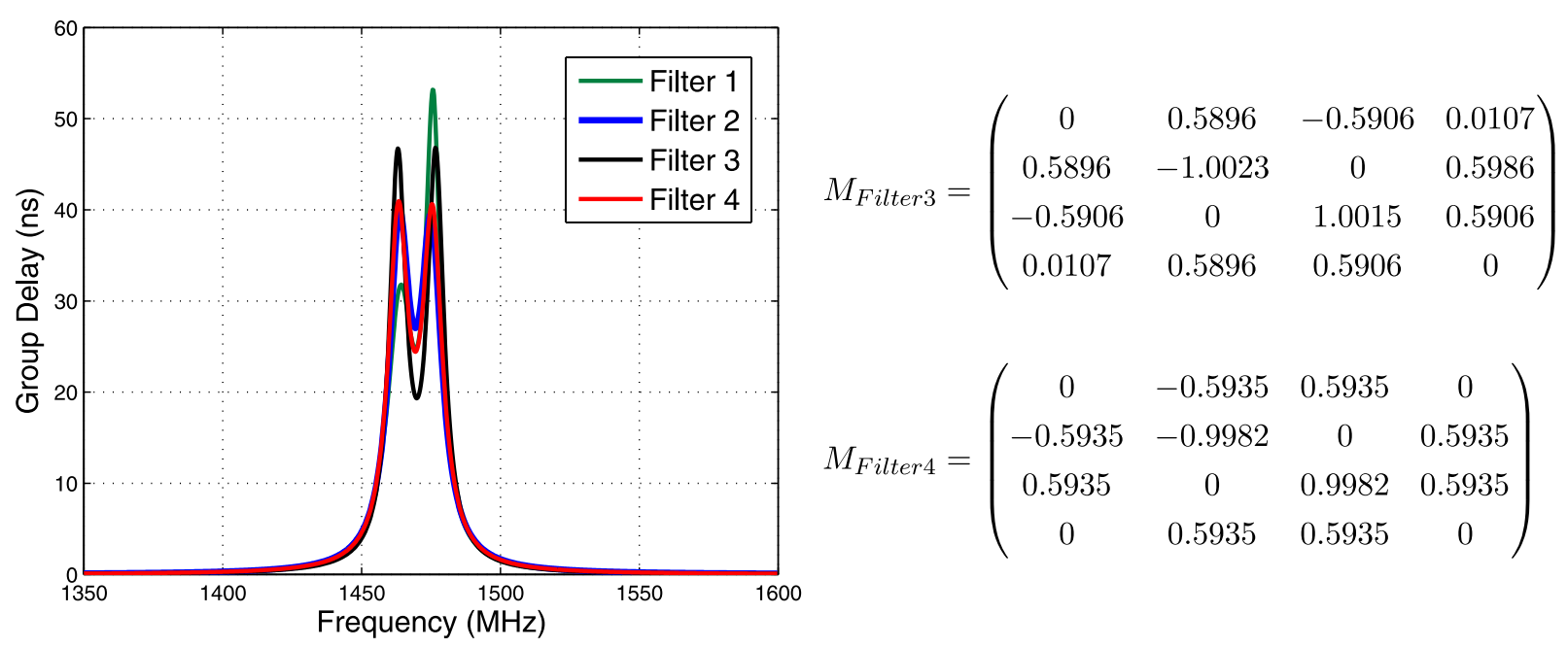

(b)

Figure 3. Different responses of the modified doublet topology (poles at 1.465 and $1.475 \mathrm{GHz}$ ). Filter 1: complex transmission zeros above the passband (placed at $1.50 \mathrm{GHz}$, with real part $\delta=3$ ); Filter 2: complex transmission zeros centered in the passband (placed at $1.47 \mathrm{GHz}$, with real part $\delta=3$ ); Filter 3: real transmission zeros (placed at 1.42 and $1.52 \mathrm{GHz}$ ), pseudoelliptic response; Filter 4: Chebyshev response. Coupling matrices of all filters are given as reference. (a) Scattering parameters. (b) Group delay. 
Table 1. Maximum Variation of the Group Delay Within the Passband for the Responses Shown in Figure 3b

\begin{tabular}{lcccc}
\hline & Filter 1 & Filter 2 & Filter 3 & Filter 4 \\
\hline Difference, ns & 22.19 & 12.68 & 27.49 & 16.54 \\
\hline
\end{tabular}

maximum number of transmission zeros for increased selectivity.

[8] In addition to the theoretical discussion, several filter prototypes implementing different frequency responses are manufactured and tested. Measured results are found to be in good agreement with respect to predictions, therefore validating the new filtering structures.

\section{Theory}

[9] It can be seen that the coupling scheme shown in Figure 1 corresponds to a transversal filter. In particular, there are no direct couplings between resonators, and each input/output port is directly coupled to both resonators. With respect to the coupling schemes originally proposed by Rosenberg and Amari [2003], Figure 1 introduces an additional direct coupling $\left(M_{\mathrm{SL}}\right)$ between the ports shown with dashed line [Amari and Rosenberg, 2003]. General synthesis techniques for these filters were presented by Cameron [2003] and Amari and Rosenberg [2003].

[10] If the direct coupling between ports is not introduced, then only one transmission zero can be placed at finite frequencies. The transmission zero can easily be shifted from one side of the passband to the other side, by changing the signs of the coupling matrix entries, as shown in Figure 2. This was recognized by Rosenberg and Amari [2003] as the zero shifting property of these transversal structures. Physically, the presence of this transmission zero can be explained as a phase cancellation that occurs between the signals in both resonators. The input signal is split into two paths (see Figure 1). The signals in the two paths suffer different phase changes, because of the different electrical lengths of the resonators. When the phase difference equals $180^{\circ}$, a destructive interference occurs, thus producing the transmission zero. This $180^{\circ}$ phase shift difference is also related to the negative sign in one of the four couplings shown in solid line in Figure 1. This negative coupling can also be seen in the coupling matrices included in Figure 2.

[11] The alternative coupling scheme introduced in Figure 1 [Amari and Rosenberg, 2003], also benefits from all these properties. In addition, the introduction of a new coupling between the ports produces a second transmission zero. The most typical response for this topology will be the synthesis of pseudo-elliptic filters. This second transmission zero can be used to obtain high selectivity at both sides of the passband. To do this, the resonator that changes sign from input to output must be tuned below the center frequency of the filter. Alternatively, both zeros can be shifted to the same side of the passband. To do this, the resonator that changes sign must be tuned above the center frequency of the filter. In this last case, the proper adjustment of the couplings can place the transmission zeros in the complex plane. All these different responses can be seen in Figure 3. Filter 3 in the legend has two transmission zeros in the real axis, while Filter 1 and Filter 2 have a pair of complex transmission zeros. The figures also include Filter 4, whose transmission zeros are located at infinity (Chebyschev response).

[12] We can observe from the results obtained in Figure $3 \mathrm{a}$ that the reflection parameter is almost the same in all cases. As expected, it can be seen that greater selectivity in the transmission response has the drawback of a larger variation in the group delay. We also see that it is possible to achieve phase equalization by placing the transmission zeros in the complex plane. In particular, the maximum variation of the group delay inside the passband for the filters shown in Figure $3 \mathrm{~b}$ are compared in Table 1.

[13] It can be seen that the filter with less variation in the group delay corresponds to Filter 2, with transmission zeros in the complex plane centered in the passband. Next, the module of the transmission parameter and the value of the group delay are compared depending on the real part of the complex transmission zeros. It can be seen in Figure 4 the trade off between selectivity and phase equalization. The maximum variation of the group delay within the passband is collected in Table 2, where Filter B corresponds to Filter 2 in Figure 3. The coupling matrices for all three filters are also included in Figure 4 for reference.

\section{Implementation}

[14] In this section we present several compact implementations of the coupling topology shown in Figure 1, in microstrip technology. For the resonators we have chosen either open-loop resonators of lengths $(\lambda / 2)$ and $(\lambda)$, or a short-circuited stub of length $(\lambda / 4)$. The differences in resonant frequency between the two resonators with respect to the center frequency of the filter, lead to the non zero entries in the coupling matrix diagonal.

[15] The coupling between the input/output ports and the resonators (solid line in Figure 1) is accomplished by coupled lines in a shunt configuration, as it can be seen in Figure 5a. Besides, an additional direct coupling between the ports is needed (dashed line represented in Figure 1). The implementation of this last coupling in microstrip technology can be accomplished by extending and bending the input and output lines, as it can be seen in Figure 5a. Figure 5b shows a typical frequency 


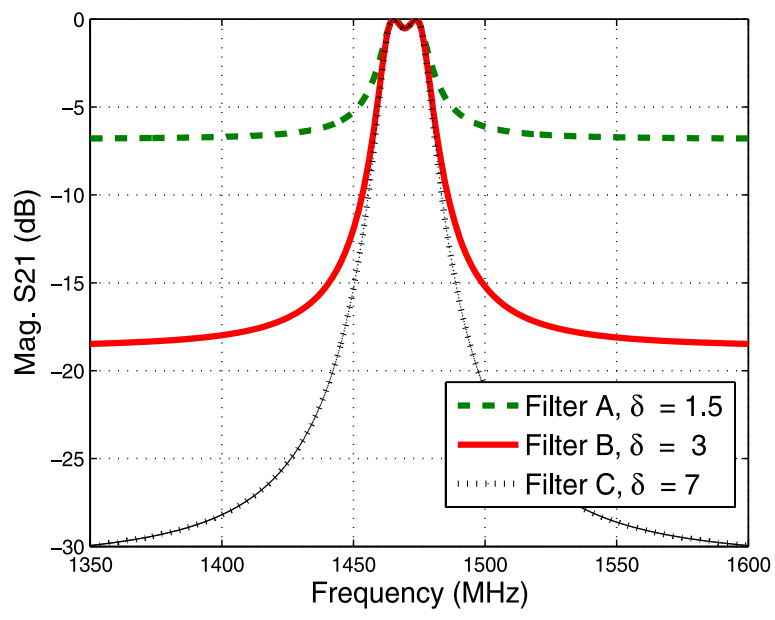

$$
\begin{aligned}
& M_{\text {FilterA }}=\left(\begin{array}{cccc}
0 & 0.6440 & -0.6440 & 0.2409 \\
0.6440 & 0.8763 & 0 & 0.6440 \\
-0.6440 & 0 & -0.8763 & 0.6440 \\
0.2409 & 0.6440 & 0.6440 & 0
\end{array}\right) \\
& M_{\text {FilterB }}=\left(\begin{array}{cccc}
0 & -0.6104 & 0.6104 & 0.0581 \\
-0.6104 & -0.9756 & 0 & 0.6104 \\
0.6104 & 0 & 0.9756 & 0.6104 \\
0.0581 & 0.6104 & 0.6104 & 0
\end{array}\right)
\end{aligned}
$$

(a)

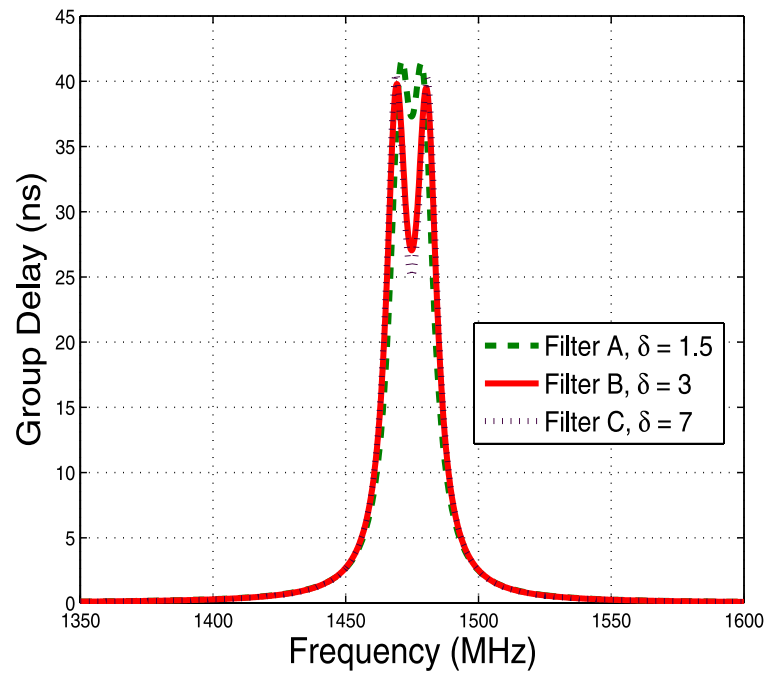

$$
M_{\text {FilterC }}=\left(\begin{array}{cccc}
0 & 0.5979 & -0.5979 & 0.0142 \\
0.5979 & 0.9930 & 0 & 0.5979 \\
0.5979 & 0 & -0.9930 & 0.5979 \\
0.0142 & 0.5979 & 0.5979 & 0
\end{array}\right)
$$

(b)

Figure 4. Electrical characteristics of several filters with complex transmission zeros in the center of the passband. The direct coupling $M_{\mathrm{SL}}$ must be increased to reduce the real part of the complex roots and improve equalization ( $\delta$ stands for the real part of the complex roots). (a) $\left|S_{21}\right|$ parameter. (b) Group delay. 
Table 2. Maximum Variation of the Group Delay Within the Passband for the Responses Shown in Figure 4

\begin{tabular}{lccr}
\hline & Filter A & Filter B & Filter C \\
\hline Difference, ns & 4.3 & 12.68 & 15.56 \\
\hline
\end{tabular}

response of this structure. Its coupling matrix can be obtained easily with the technique reported by Cameron [2003]:

$$
M=\left(\begin{array}{cccc}
0 & 0.4184 & -1.1147 & 0.1028 \\
0.4184 & -1.3528 & 0 & 0.4184 \\
-1.1147 & 0 & 1.2928 & 1.1147 \\
0.1028 & 0.4184 & 1.1147 & 0
\end{array}\right)
$$

For this structure, the direct coupling controls the proximity of the transmission zeros to the passband. When this direct coupling is increased, both transmission zeros approach the passband of the filter. To produce the transmission zeros at both sides of the passband, the open-loop resonator has to be tuned above the shortcircuited resonator. In this way, the transmission zeros are combined for increased selectivity at both sides of the passband. If on the contrary we tune the open-loop resonator below the short-circuited resonator, then both zeros will shift to one side of the passband, as can be seen in Figure 6. For this last structure we have selected $l_{2}=4.9 \mathrm{~mm}$, while the other dimensions are the same as in Figure 5a.

[16] The direct capacitive coupling introduced by the bent stub depends on two parameters, namely the gap $\left(d_{\mathrm{g}}\right)$ and the coupling length $\left(l_{c}\right)$ (see Figure 5a). To see how this element can synthesize a given coupling, the direct $M_{\mathrm{SL}}$ coupling is presented in Figure 7 as a function of the length $\left(l_{c}\right)$, for different values of the gap $\left(d_{\mathrm{g}}\right)$. The maximum allowed stub length for the design in Figure 5a, is $l_{c}=5.9 \mathrm{~mm}$. This maximum value is due to the physical space existing between the two resonators. It can be seen in Figure 5a, that the empty space left by the open-loop resonator is used to place the coupling bent stub. This results in a very compact structure. For issues concerning the practical realization of the filter, the minimum gap is set to $d_{\mathrm{g}}=0.1 \mathrm{~mm}$. With these limits, the maximum direct coupling that can be obtained with this configuration is of about: $M_{\mathrm{SL}}=0.34$.

Table 3. Dimension $L_{\mathrm{o}}$ for the Filters Shown in Figure 11, and Maximum Variation of the Group Delay Within the Passband

\begin{tabular}{lccc}
\hline & Filter 1 & Filter 2 & Filter 3 \\
\hline$M_{\mathrm{SL}}=M_{\mathrm{LS}}$ & 0.0492 & 0.0886 & 0.024 \\
Length $L_{\mathrm{o}}, \mathrm{mm}$ & 3.8 & 2.3 & 5 \\
Difference, ns & 18.64 & 21.26 & 17.95 \\
\hline
\end{tabular}

[17] An alternative structure that implements the same coupling scheme shown in Figure 1 is presented in Figure $8 \mathrm{a}$. In this case, the through line from input to output implements a direct coupling of inductive type between the input/output ports. The response of this structure is presented in Figure $8 \mathrm{~b}$, showing a band-stop response with two reflection zeros.

[18] The results shown in Figure 8 correspond to a stopband filter with a sharp transition at both sides of the stopband. The method for the synthesis of stop-band transfer functions was derived by Amari and Rosenberg

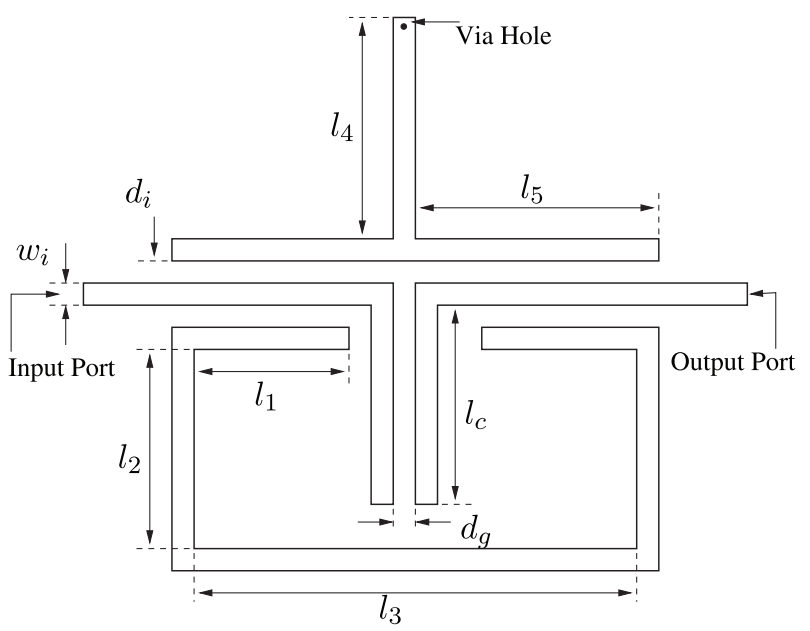

(a)

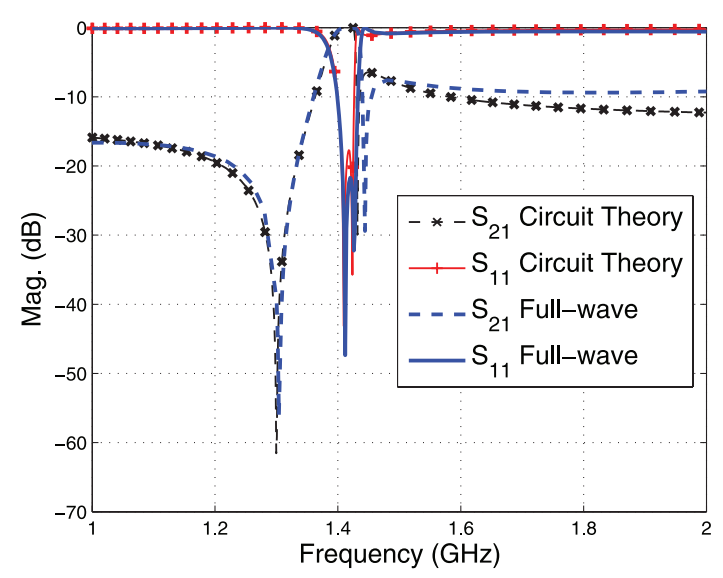

(b)

Figure 5. Bandpass filter implementing the coupling topology shown in Figure 1. Dimensions are $w_{i}=1.8 \mathrm{~mm}$, $d_{i}=0.3 \mathrm{~mm}, l_{1}=8.0 \mathrm{~mm}, l_{2}=4.1 \mathrm{~mm}, l_{3}=21.5 \mathrm{~mm}$, $l_{4}=8.2 \mathrm{~mm}, l_{5}=11.35 \mathrm{~mm}, l_{c}=5.5 \mathrm{~mm}$, and $d_{\mathrm{g}}=$ $0.3 \mathrm{~mm}$. (a) Geometry of the filter. (b) Scattering parameters of the filter. 


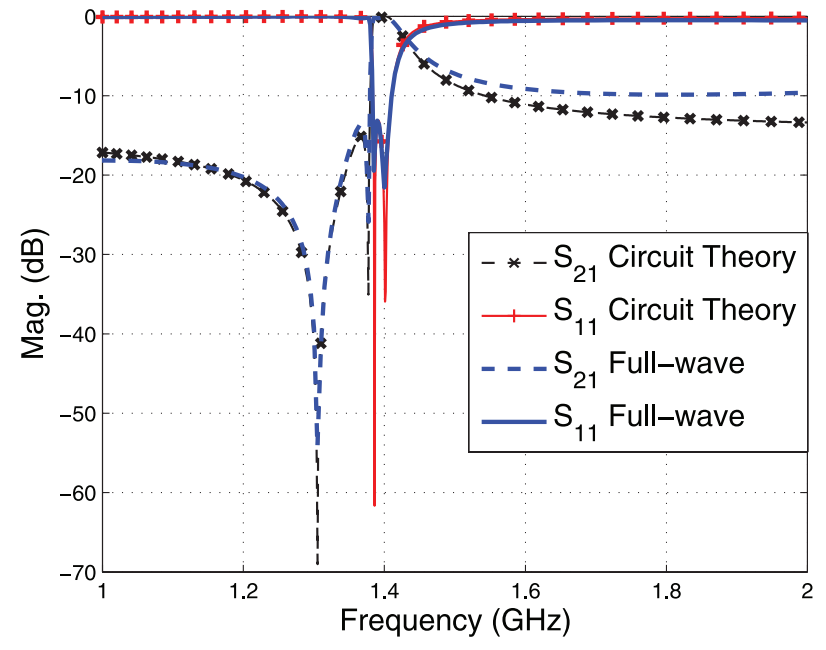

Figure 6. S-parameters of a transversal filter implementing two transmission zeros below the passband. Response obtained changing the value of $l_{2}$ of Figure $5 \mathrm{a}$ to $4.9 \mathrm{~mm}$.

[2004b]. Following this procedure the final coupling matrix results to be:

$$
M=\left(\begin{array}{cccc}
0 & -1.8682 & 0.9421 & -1.0445 \\
-1.8682 & 1.5941 & 0 & 1.8682 \\
0.9421 & 0 & 1.0550 & 0.9421 \\
-1.0445 & 1.8682 & 0.9421 & 0
\end{array}\right)
$$

This structure presents a dual frequency behavior as compared to the previous one. The direct coupling here

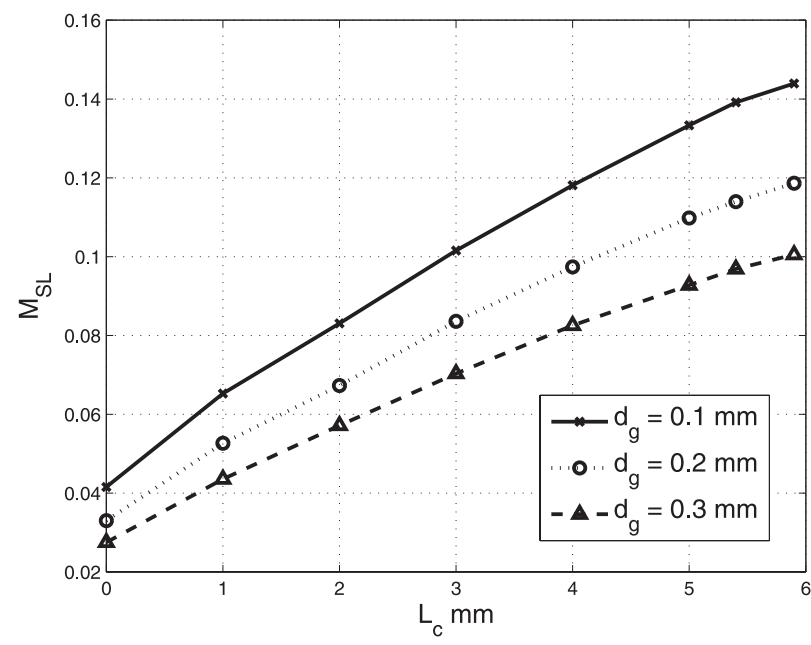

Figure 7. Coupling characteristics of the bent stub element.

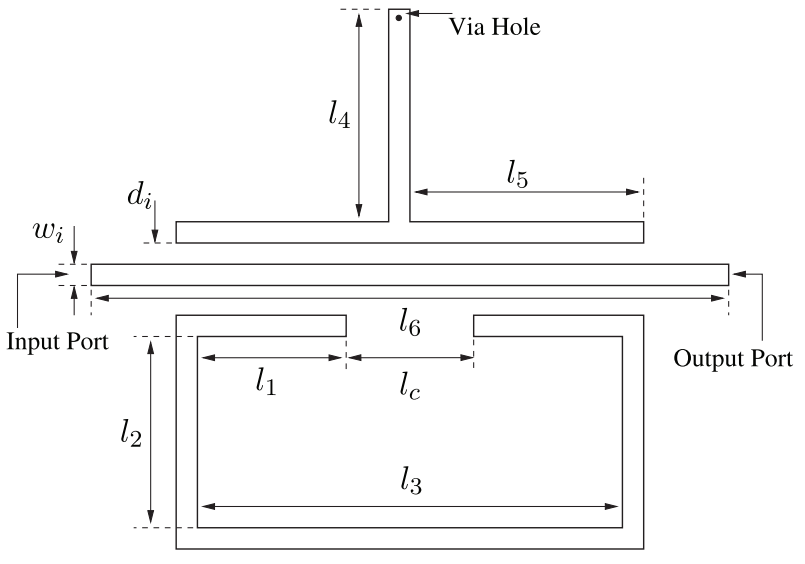

(a)

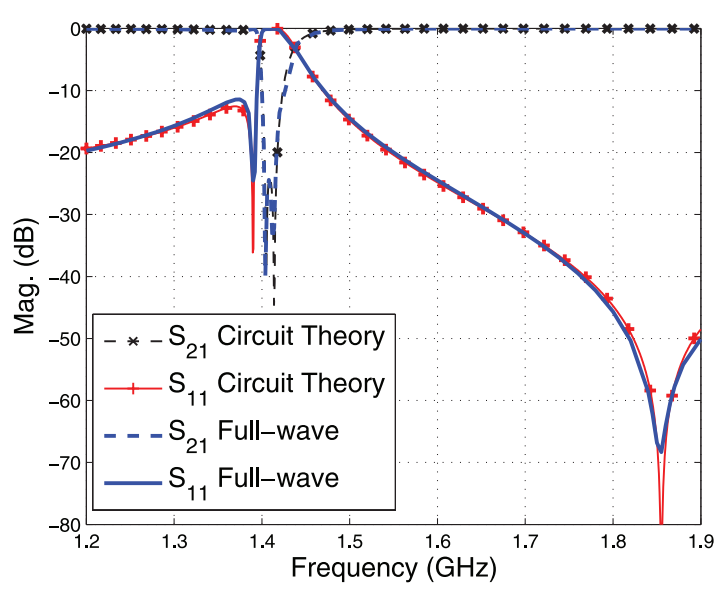

(b)

Figure 8. Band-stop filter implementing the coupling topology shown in Figure 1. Dimensions are $w_{i}=1.8 \mathrm{~mm}$, $d_{i}=0.3 \mathrm{~mm}, l_{1}=8.0 \mathrm{~mm}, l_{2}=4.4 \mathrm{~mm}, l_{3}=21.5 \mathrm{~mm}, l_{4}=$ $8.0 \mathrm{~mm}, l_{5}=11.35 \mathrm{~mm}, l_{c}=5.5 \mathrm{~mm}$, and $l_{6}=45 \mathrm{~mm}$. (a) Geometry of the filter. (b) Scattering parameters of the filter.

controls the proximity of the reflection zeros to the stopband. This direct coupling can be modified by varying the width of the line joining both ports. For instance, the direct coupling $\left(M_{\mathrm{SL}}\right)$ can be increased by widening the central connecting area as shown in the inset of Figure 9. By doing this, the reflection zeros approach the stop-band, as also shown in the results presented in the same Figure 9. The coupling matrices of the different responses are also presented in Figure 9 for reference. We can observe that the direct coupling 


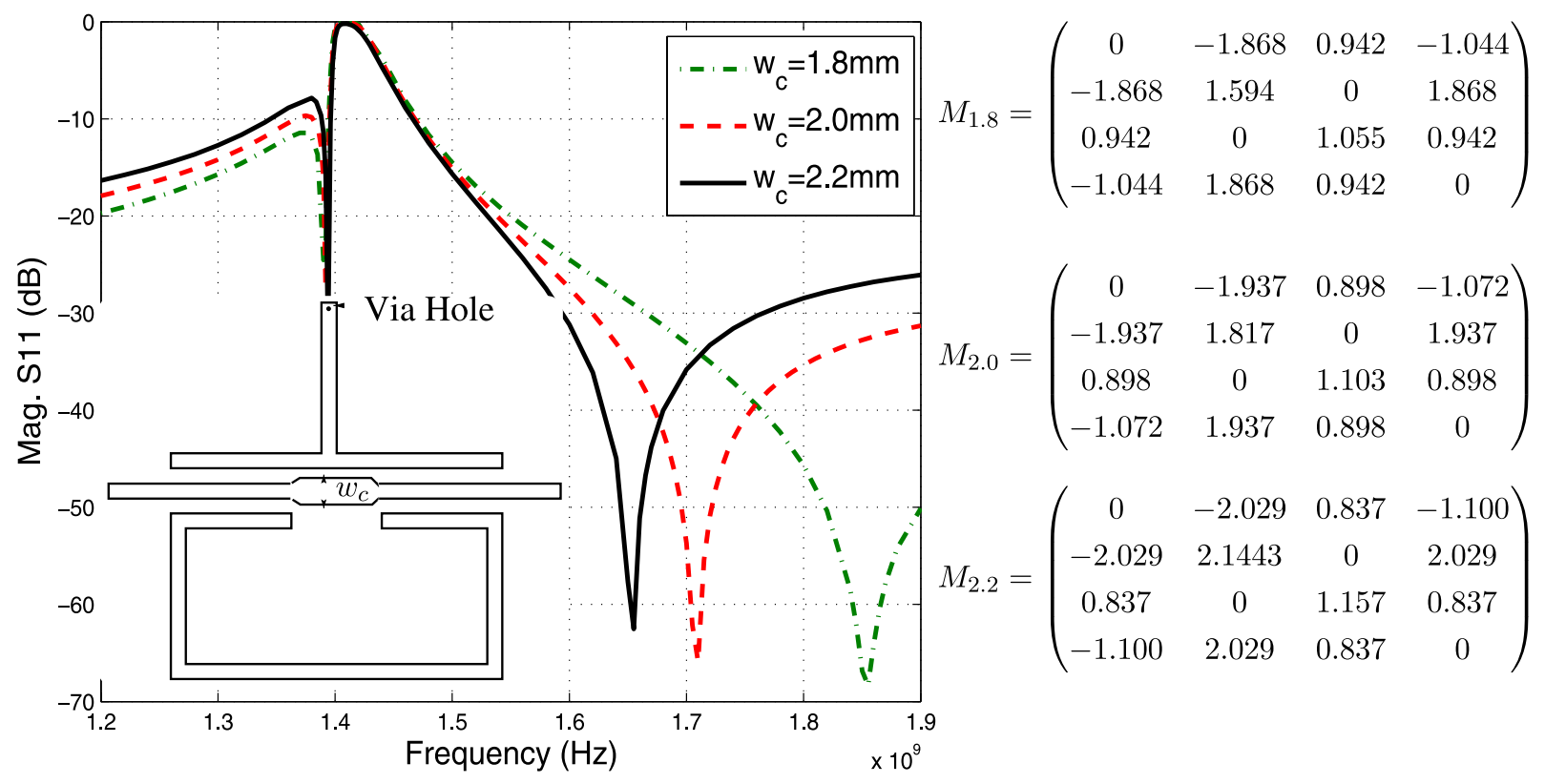

Figure 9. Reflection parameter of a band-stop filter for different values of $w_{\mathrm{c}}$ (shown in the inset of the figure). All other dimensions are the same as in Figure 8. By increasing the direct coupling $M_{\mathrm{SL}}$, the reflection zeros can be placed closer to the stop band.

increases from $M_{\mathrm{SL}}=-1.044\left(\right.$ when $\left.w_{\mathrm{c}}=1.8 \mathrm{~mm}\right)$ to $M_{\mathrm{SL}}=-1.100$ (when $w_{\mathrm{c}}=2.2 \mathrm{~mm}$ ).

[19] Finally, the last practical implementation presented in this paper corresponds to a band-pass filter with transmission zeros in the complex plane. This time we have used two open-loop resonators to implement the filter. To synthesize the sign change required in one of the elements of the coupling matrix, the first line is tuned at the $(\lambda / 2)$ resonance, while the second line is tuned around the $(\lambda)$ resonance. Moreover, to place both zeros on only one side of the passband, the shorter resonator has to be tuned above the longer resonator. The direct coupling between the ports follows the same principle described before. A possible layout for this structure can be seen in Figure 10.

[20] The coupling matrix of the "Filter 1" shown in the legend of Figure 11 can be easily obtained as:

$$
M=\left(\begin{array}{cccc}
0 & -0.5471 & 0.6668 & 0.0492 \\
-0.5471 & -0.9317 & 0 & 0.5471 \\
0.6668 & 0 & 0.9096 & 0.6668 \\
0.0492 & 0.5471 & 0.6668 & 0
\end{array}\right)
$$

Moreover, the different responses presented in Figure 11 have been obtained by varying the value of the coupling matrix corresponding to the input/output coupling $M_{\mathrm{SL}}$. We can observe that by changing this coupling, the transmission zeros can be moved from the real axis
(Filter 2 in the legend) to the complex plane (Filter 1 and Filter 3 in the legend). The adjustment of this coupling can be done by modifying the length $L_{\mathrm{o}}$ shown in Figure 10. The responses shown in Figure 11 have been

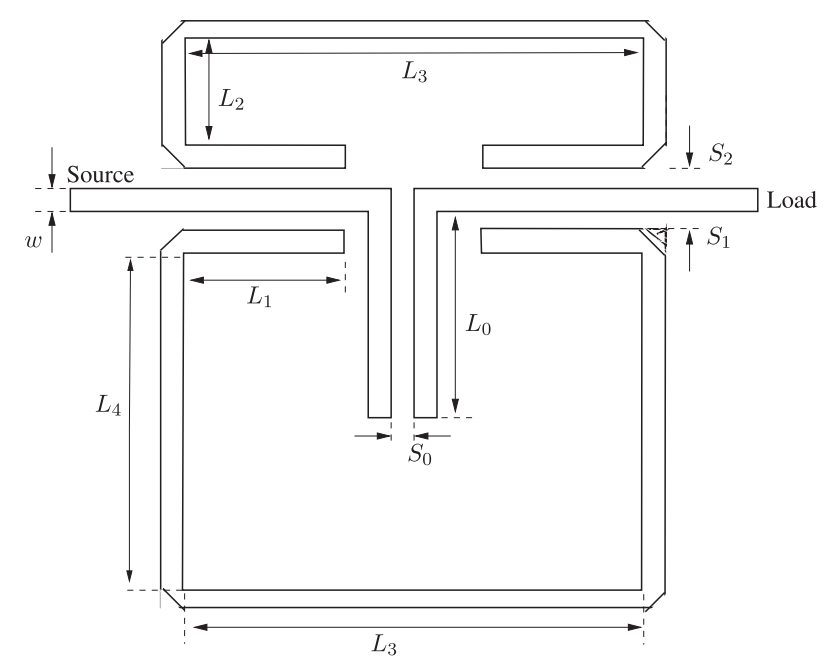

Figure 10. Implementation of a band-pass filter following the coupling scheme in Figure 1, with two transmission zeros in the complex plane. Dimensions are $w=1.8$

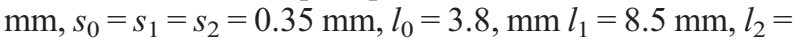
$3.0 \mathrm{~mm}, l_{3}=21.9 \mathrm{~mm}$, and $l_{4}=26.5 \mathrm{~mm}$. 


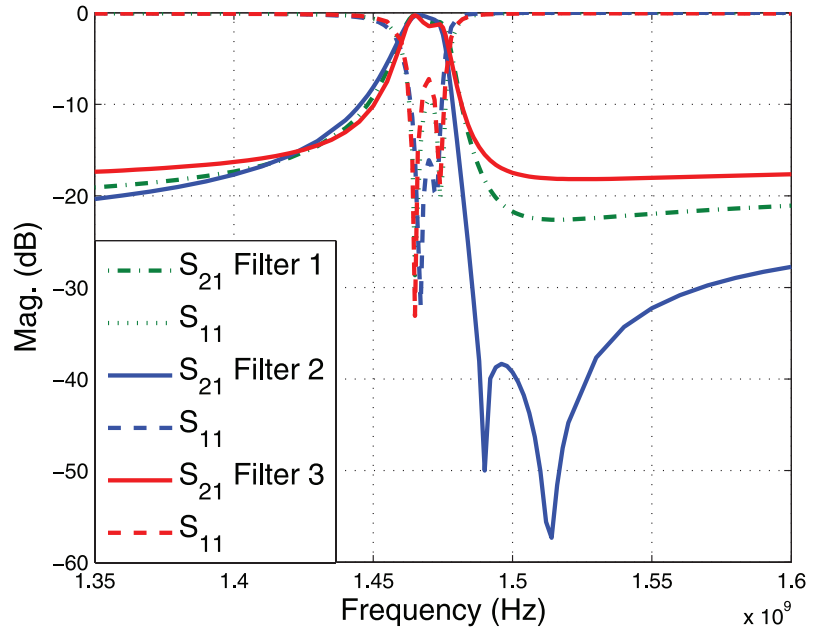

(a)

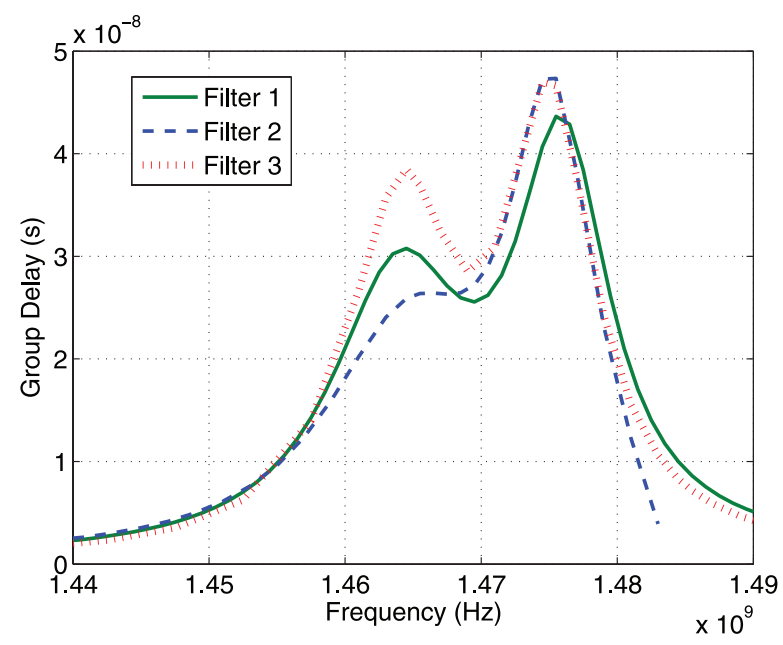

(b)

Figure 11. Different filters with two transmission zeros above the passband. Filter 1: two complex transmission zeros on the right side of the passband (placed at $1.48 \mathrm{GHz}$, with real part $\delta=3.2$; dimensions in Figure 10); Filter 2: two transmission zeros in the real axis above the passband (placed at 1.49 and $1.51 \mathrm{GHz}$ ); Filter 3: similar to Filter 1 but with smaller real part of the complex roots $(\delta=2.2)$. (a) Scattering parameters. (b) Group delay.

obtained with the lengths given in Table 3 . This table also shows the variation of the group delay inside the passband, demonstrating the equalization effect of complex transmission zeros. The value of the direct coupling $M_{\mathrm{SL}}$ is also shown for reference.

\section{Results}

[21] The filters shown in Figure 5a, Figure 8a and "Filter 1" of Figure 11 have been manufactured and tested. The substrate selected for manufacturing is an RTDuroid/6006, with relative permittivity $\varepsilon_{r}=6.15$, and thickness $1.27 \mathrm{~mm}$. Figure 12 and Figure 13 compare the measured results with predictions obtained by full-wave simulations based on an Integral Equation technique. In the simulations, losses are included in both, the dielectric substrate $(\tan (\delta)=0.0019)$ and in the printed metallic areas $\left(\sigma=410^{7} \Omega^{-1} / \mathrm{m}\right)$. The circuits have been

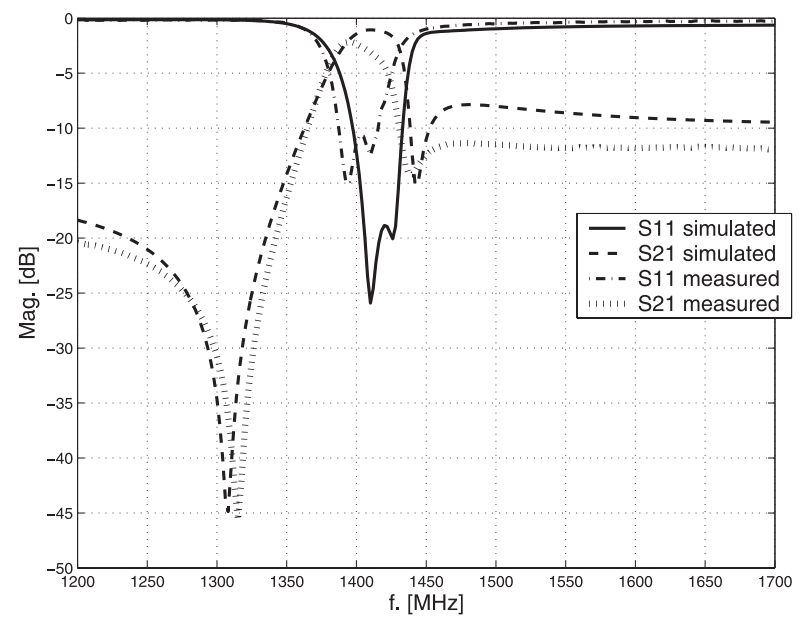

(a)

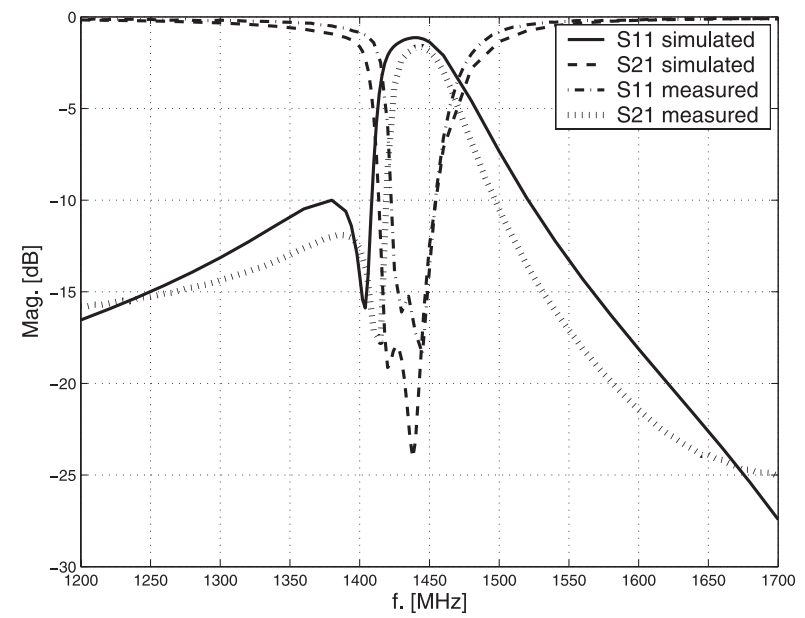

(b)

Figure 12. Measured versus simulated results for the manufactured filters. (a) Bandpass filter shown in Figure 5a. (b) Bandpass filter shown in Figure 8. 


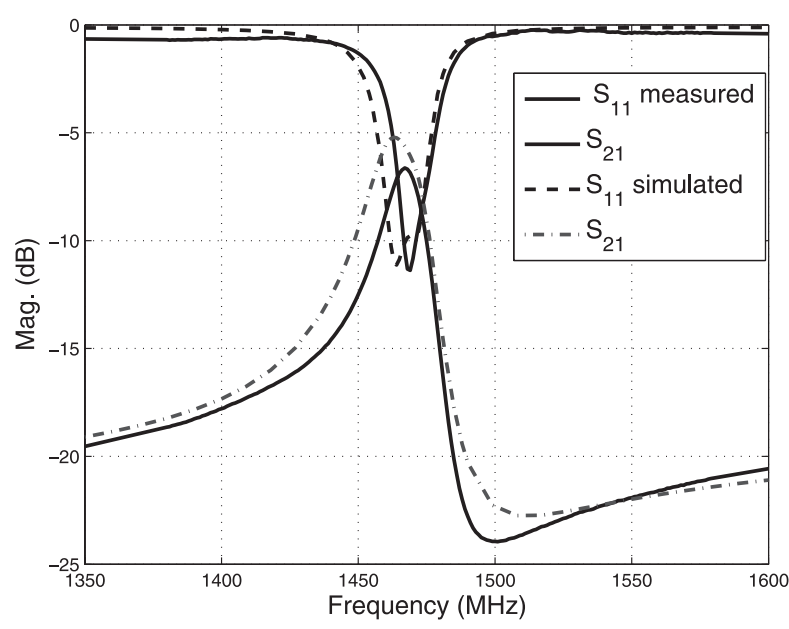

(a)

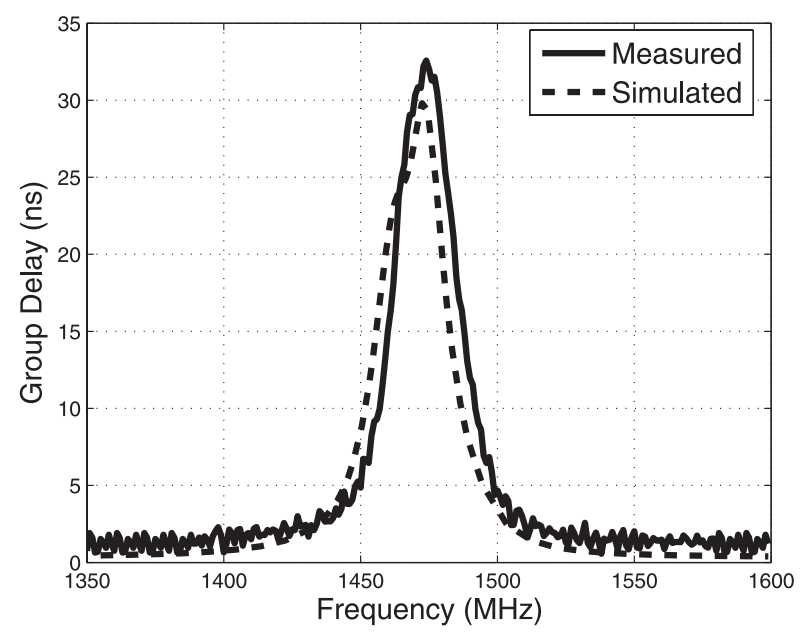

(b)

Figure 13. Measured versus simulated results for the band-pass filter with complex zeros shown in Figure 10. (a) Scattering parameters. (b) Group delay.

manufactured with an LPKF Protomat drilling machine, and measurements are taken with an HP-8714-ES vector network analyzer. It can be observed good agreement between measured results and predictions. Only there are some differences in the return loss performance shown in Figure 12a. This is because of the high sensitivity of the return losses in this type of filters, to the lengths of the resonators. We have observed that a variation in the length of the longer resonator, in only $0.1 \mathrm{~mm}$, produces a large variation in the return losses from $-18 \mathrm{~dB}$ to $-10 \mathrm{~dB}$.
[22] The first filter shows a $3.7 \%$ relative bandwidth, and $1.5 \mathrm{~dB}$ of insertion losses inside the passband. The band-stop filter shows a $1.9 \%$ relative bandwidth, and $1.5 \mathrm{~dB}$ of reflection losses inside the stop-band. The insertion losses far from the stop-band amounts only $0.15 \mathrm{~dB}$. The third filter presents higher insertion looses, because of the small bandwidth of the filter (less than $1 \%)$. We can clearly observe in the results the presence

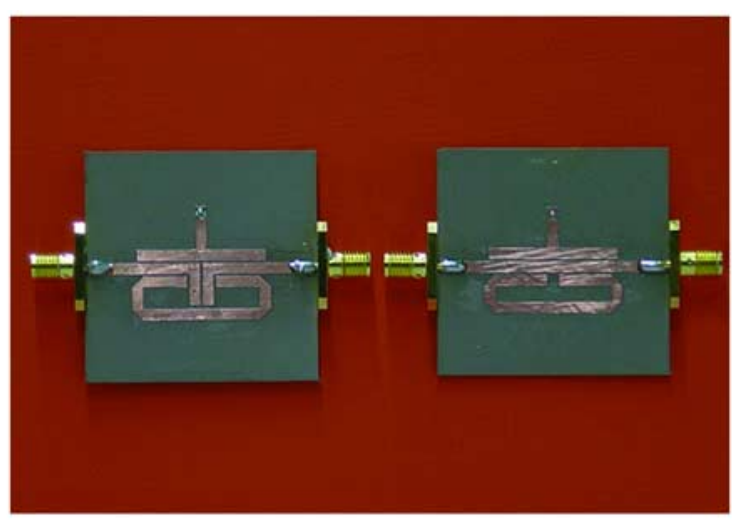

(a)

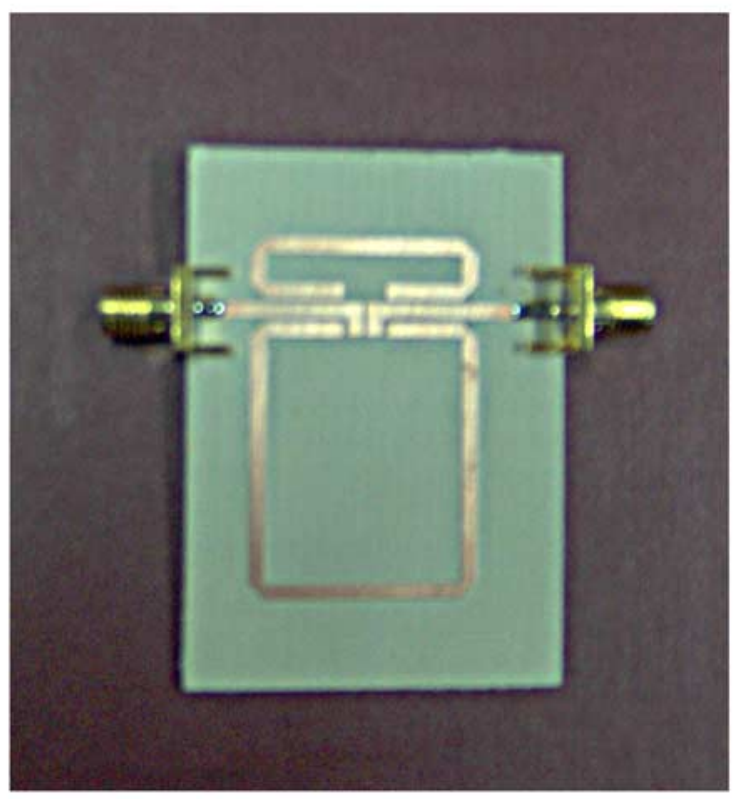

(b)

Figure 14. Aspect of the real manufactured filters. (a) Bandpass and band-stop filters. (b) Filter with complex transmission zeros. 
of the complex zeros slightly above the passband. These are good features for the manufactured filters, and validates the new structures for practical applications. The final manufactured prototypes are shown in Figure 14.

\section{Conclusions}

[23] This paper has proposed novel structures to implement microwave filters. The topology implemented is based on a transversal filter, where direct coupling between the ports is introduced. This coupling produces a new transmission zero, which can be used to synthesize quasi-elliptic responses for maximum selectivity on both sides of the passband. Other responses are easily obtained, such as band-stop filters or filters with group delay equalization. On the basis of the new coupling schemes, three compact implementations of microwave filters are proposed in microstrip technology. In the first structure, the coupling between the ports is of capacitive type, and it is introduced with the help of a bent stub. The coupling capabilities of this element allow to place the transmission zeros close to the passband. The second structure changes the capacitive coupling to an inductive one, and synthesizes a band-stop filter with two reflection zeros. The last structure shifts the location of the transmission zeros to one side of the passband, implementing two transmission zeros in the complex plane for phase equalization. Measured results are in good agreement with respect to predictions, and validate the structures for practical applications.

[24] Acknowledgments. This work has been developed with support from the Spanish National Project (CICYT) with Ref. TEC2007-67630-C03-02 and the Regional Seneca Project with Ref. 02972/PI/05.

\section{References}

Amari, S., and U. Rosenberg (2003), A universal building block for advanced modular design of microwave filters, IEEE Microwave Wireless Components Lett., 13(12), 541-543.

Amari, S., and U. Rosenberg (2004a), Synthesis and design of novel in-line filters with one or two real transmission zeros, IEEE Trans. Microwave Theory Tech., 52(5), 1464-1478.
Amari, S., and U. Rosenberg (2004b), Direct synthesis of a new class of bandstop filters, IEEE Trans. Microwave Theory Tech., 52(2), 607-616.

Cameron, R. J. (2003), Advanced coupling matrix synthesis techniques for microwave filters, IEEE Trans. Microwave Theory Tech., 51(1), 1-10.

Cameron, R. J., and J. D. Rhodes (1981), Asymmetric realizations for dual-mode bandpass filters, IEEE Trans. Microwave Theory Tech., 29(1), 51-58.

Hong, J. S., and M. J. Lancaster (2000), Design of highly selective microstrip bandpass filters with a single pair of attenuation poles at finite frequencies, IEEE Trans. Microwave Theory Tech., 48(7), 1098-1107.

Kurzok, R. M. (1966), General four-resonators filters at microwave frequencies, IEEE Trans. Microwave Theory Tech., 14(7), 295-296.

Lee, J., M. S. Uhm, I. B. Yom, S. P. Lee, and C. K. Choi (2003), Simultaneous reduction of amplitude and group delay variation of input demultiplexer for ku-band satellite transponder, Proc. IEEE Vehicular Technology Conf., 4, 2669-2674, VTC'03-Fall.

Moktaari, M. K. R., J. Bornemann, and S. Amari (2006), Coupling-matrix design of dual and triple passband filters, IEEE Trans. Microwave Theory Tech., 54(11), 3940-3946.

Rebenaque, D. C., F. Q. Pereira, J. P. Garcia, A. A. Melcon, and M. Guglielmi (2004), Two compact configurations for implementing transmission zeros in microstrip filters, IEEE Microwave Wireless Components Lett., 14(10), 475-477.

Rosenberg, U., and S. Amari (2003), Novel coupling schemes for microwave resonator filters, IEEE Trans. Microwave Theory Tech., 50(12), 2896-2902.

Williams, A. E. (1970), A four-cavity elliptic waveguide filter, IEEE Trans. Microwave Theory Tech., 18(12), 1109-1114.

A. Álvarez-Melcón, D. Cañete Rebenaque, J. L. GómezTornero, M. Martínez-Mendoza, and J. Pascual-García, Departamento de Tecnologías de la Información y las Comunicaciones, Technical University of Cartagena, UPCT, Campus Muralla del Mar. Plaza del Hospital, 1 E-30202 Cartagena, Murcia, Spain. (david.canete@upct.es) 